\title{
HIGHLIGHTS
}

SEXUAL DYSFUNCTION

\section{Therapeutic benefit of pentoxifylline for Peyronie's disease}

A double-blind, placebo-controlled randomized trial of oral pentoxifylline for men with early chronic Peyronie's disease has demonstrated modest improvements in penile curvature, plaque size and erectile function. Results of the trial were reported by a team of researchers from Sharid Beheshti University in Tehran, who suggest that pentoxifylline might be a feasible treatment option-in combination with topical and intralesional drugs - for affected individuals who would rather not undergo surgery.

Peyronie's disease affects about $6 \%$ of middle-aged men and is characterized by fibrosis of the tunica albuginea, causing painful erection, penile curvature and erectile dysfunction. Pentoxifylline is a nonselective phosphodiesterase inhibitor thought to have anti-inflammatory and antifibrogenic activity. It has been used widely to treat other fibrotic disorders, which led the research team to propose that it might be an effective treatment for Peyronie's disease.

Safarinejad et al. randomized

228 patients with early chronic disease to receive either $400 \mathrm{mg}$ sustained-release pentoxifylline or placebo twice daily for 6 months. Individuals were assessed for both subjective and objective changes every month after receiving the initial dose until 1 month after completion of treatment. Beneficial effects of treatment were noted 2 months into the study, and steadily increased during the treatment period.

Overall, significantly fewer patients on pentoxifylline experienced disease progression (defined as an increase in plaque volume, penile curvature, penile pain or end-diastolic velocity, or a decrease in resistivity index, peak systolic velocity or International Index of Erectile Function [IIEF] score) than those who received placebo (11\% versus $42 \%$ ). Furthermore, $37 \%$ of men in the treatment group reported an improvement in penile curvature and plaque volume compared to only $5 \%$ of patients given placebo. Most noticeably, at the end of the study the IIEF total score was markedly higher in the pentoxifylline $\operatorname{arm}(P=0.02)$.

The safety of pentoxifylline was also demonstrated; only one patient stopped taking the medication owing to adverse effects. No serious adverse effects were noted, and just $5 \%$ of treated individuals reported nausea, vomiting or dyspepsia.

The authors suggest that further studies are warranted to determine the potential role of this drug. They hope this trial will encourage future investigative efforts regarding the effectiveness of nonsurgical therapies for Peyronie's disease.

Sarah Payton

Original article Safarinejad, M. R. et al.A double-blind placebo-controlled study of the efficacy and safety of pentoxifylline in early chronic Peyronie's disease. BJU Int. doi:10.1111/j.1464-410X.2009.09041.x 